\title{
Meiotic roles of Mec1, a budding yeast homolog of mammalian ATR/ATM
}

\author{
Jesús A. Carballo \& Rita S. Cha* \\ Division of Stem Cell Biology and Developmental Genetics, MRC National Institute for Medical Research, \\ The Ridgeway, Mill Hill, London, NW7 1AA, UK; Tel: 44 (0)208 816 2243; Fax: 44 (0)208 816 2534; \\ E-mail:rcha@nimr.mrc.ac.uk \\ *Correspondence
}

Key words: chromosome synapsis, Mec1, meiosis, meiotic progression, meiotic recombination

\begin{abstract}
Budding yeast Mec1, a homolog of mammalian ATR/ATM, is an essential chromosome-based signal transduction protein. Mec1 is a key checkpoint regulator and plays a critical role in the maintenance of genome stability. Mec1 is also required for meiosis; loss of Mec1 functions leads to a number of meiotic defects including reduction in recombination, loss of inter-homolog bias, loss of crossover control, and failure in meiotic progression. Here we review currently available data on meiotic defects associated with loss of Mec1 functions and discuss the possibility that Mec1 may participate as a fundamentally positive player in coordinating and promoting basic meiotic chromosomal processes during normal meiosis.
\end{abstract}

\section{Introduction}

Mec1 belongs to the evolutionarily conserved ATM/ ATR family of signal transduction proteins whose members include mammalian ATR and ATM, Drosophila Mei-41, fission yeast $\mathrm{rad} 3+$ and tel1+, and budding yeast Mec1 and Tel1. They are chromosome-associated proteins, best known for their roles in the DNA damage response where they sense the presence of damage and transmit appropriate signals that trigger cell cycle arrest. Additionally, the ATM/ ATR family of proteins play essential roles in the repair of DNA damage by regulating the activity of proteins that participate in the repair process. As expected, inactivation of these proteins confers sensitivity to a wide array of genotoxic agents (Zhou \& Elledge 2000).

Interestingly, however, loss of Mec1, ATR, or ATM functions also leads to profound defects during the unchallenged life of the cell, indicating that their functions are required even in the absence of any exogenously induced damage (Kato \& Ogawa 1994, $\mathrm{Xu}$ et al. 1996). Because the nature of defects observed during unchallenged life is similar to those observed following exposure to DNA damaging agents (e.g. chromosome breakage, genome instability, and inviability), their normal functions were proposed to be analogous to those in damage response - i.e. in processing potentially lethal lesions that arise spontaneously during normal cellular life (Zhou \& Elledge 2000). However, because this view could not adequately account for a number of mecl, $A T M^{-/-}$, and $A T R^{-/-}$phenotypes, an alternative model was proposed; in this model the primary function of ATM/ATR/Mec1 during unchallenged life is in coordinating and promoting basic chromosomal events, with the observed mutant phenotypes arising from defects in these fundamental processes regardless of any failure to respond to incidental lesions (Cha \& Kleckner 2002).

The ATM/ATR family proteins also play essential roles during meiosis. In budding yeast, loss of Mec1 
functions leads to defects in meiotic recombination (Kato \& Ogawa 1994, Grushcow et al. 1999), chromosome morphogenesis (Xu et al. 1996, Grushcow et al. 1999), meiotic progression (Kato \& Ogawa 1994) and checkpoint arrest (Lydall et al. 1996). Inactivation of Mei-41 in fruit flies leads to aberrant crossover distribution (Carpenter 1979). Spermatogenesis in $A T M^{-1-}$ mice is characterized by chromosome fragmentation and apoptosis (Barlow et al. 1996, Xu et al. 1996). Because (a) ATM/ATR family proteins are best known for their checkpoint functions and (b) hundreds of programmed double-strand breaks (DSB) are introduced into the genome as a part of the meiotic program, it has been proposed that the essential meiotic role of ATM/ATR family proteins is to monitor the repair process and to delay the onset of meiosis I until the repair is complete (Hochwagen \& Amon 2006). However, as is the case for Mec1's essential role during the mitotic cell cycle, it is possible that Mec1 could play a more fundamental role during meiosis. Here we review the nature of the meiotic defects observed in mecl mutants and discuss how they could be accounted for in terms of failure in coordinating and promoting basic chromosomal events.

\section{Mec1: the functional homolog of mammalian ATM and ATR}

Inactivation of the mammalian ATM or ATR confers phenotypes that are distinguishable from one another at both the cellular and organism level. For example, whereas ATR inactivation in mice is embryonic lethal (Brown \& Baltimore 2000), $A T M^{-1-}$ mice develop pathological conditions during later stages in life (Barlow et al. 1996, Xu et al. 1996). At the cellular level, $A T R^{-1-}$ and $A T M^{-1-}$ cells show sensitivity to different genotoxic agents; $A T R^{-/-}$ cells are more sensitive to those inducing stalled replication forks or intra-strand crosslinks whereas $A T M^{-/-}$cells are more sensitive to those that induce DSB (McGowan \& Russell 2004). The ATM and ATR pathways do not appear to function completely independently of each other, and crosstalk between the two has been observed under certain conditions. For example, in facilitating a G2/M checkpoint arrest in response to DSB, ATM mediates the initial processing of the break-ends to generate replication protein A(RPA)-coated single-stranded DNA
(ssDNA), which is required for ATR recruitment and the subsequent phosphorylation and activation of Chk1 (Jazayeri et al. 2006, Cuadrado et al. 2006).

The budding yeast genome has two ATM/ATR family members, Mec1 and Tel1. In general, Mec1 is considered to be the counterpart of ATR, and Tel1 that of ATM. These correspondences are partly based on structural considerations (e.g. Perry \& Kleckner 2003) as well as on the observation that certain functions carried out by ATR are carried out by Mec1 (e.g. responding to stalled replication forks and UV damage) whereas certain other functions are primarily carried out by ATM and Tel1 (McGowan \& Russell 2004). Like mammalian ATR, Mec1 is an essential protein (Kato \& Ogawa 1994). Various hypomorphic alleles of mecl confer nearly all cellular phenotypes of $A T R^{-/-}$mutants consistent with the view that Mec1 and ATR are involved in the same cellular processes. Interestingly, mecl mutants also exhibit defects that are primarily associated with the loss of ATM function (e.g. responding to DSB; Melo et al. 2001); thus in yeast, Mec1 is also carrying out (at least some of) ATM functions. Indeed, although inactivation of Tell confers some phenotypes such as telomere shortening and a relatively small increase in gross chromosomal recombination (Greenwell et al. 1995), tell $\Delta$ mutants, by large, proliferate with growth kinetics that are indistinguishable from WT (Sanchez et al. 1996). tell $\Delta$ strains also exhibit WT resistance to different types of DNA-damaging agents (e.g. Morrow et al. 1995), indicating that the cell's repair and checkpoint functions are largely intact. Taken together, these observations suggest that budding yeast Mec1 is primarily responsible for carrying out the functions of both ATR and ATM, and that Tel1 plays a limited role. However, we cannot rule out the possibility that Mec1 and Tel1 each have a specialized set of functions, but that Mec1's roles are more differentiated in that Tel1 is unable to substitute for Mec1, whereas Mec1 can complement the functions of Tel1 effectively.

Mec1 and Tel1 show the same target specificity (below). When tested in vitro they phosphorylate many of the same proteins, including Dun1, Xrs2, and RPA (Mallory et al. 2003). Further, in all cases examined, inactivation of both Mec1 and Tel1 is required to completely abolish damage-dependent phosphorylation of their targets (e.g. Flott \& Rouse 2005, Cartagena-Lirola et al. 2006), suggesting a 
certain degree of functional overlap and/or redundancy. Nevertheless, the fact that loss of Mec1 alone is sufficient to confer lethality demonstrates that Tel1, when expressed at the physiological level, is unable to carry out the essential functions of Mec1. Since tell $\Delta$ strains proceed normally through meiosis to generate viable spores (J. Carballo, N. Hashash \& R. Cha unpublished), for the purpose of discussing normal meiotic functions, Mec1 will be considered to be the functional homolog of both ATM and ATR.

\section{Targets of Mec1}

The ATM/ATR family of proteins are serine/threonine kinases. They preferentially phosphorylate their substrates on serine or threonine residues that precede glutamine residues, so-called SQ/TQ or S/TQ motifs. Many known targets of the kinases contain SQ/TQ cluster domains or, in short, 'SCD' (Traven \& Heierhorst 2005).

One consequence of phosphorylation is regulation of the interaction of modified proteins with other proteins. Some protein-protein interactions depend on SCD phosphorylation at specific sites, whereas others occur when a certain threshold level of phosphorylated SQ/TQ motifs is achieved. In all cases examined, the phospho-threonines, rather than the phospho-serines, of phosphorylated SCD function as binding sites for the forkhead-associated (FHA) domains of other proteins. A certain level of promiscuity exists in phosphorylated SCD- and FHA domain-mediated interactions - e.g. the Rad53 (Chk2 family of kinases) SCD can interact with the FHA domain of Dun1 and Rad9, as well as the FHA domain of other Rad53 (Traven \& Heierhorst 2005).

Mec1 phosphorylates proteins involved in a wide array of cellular and chromosomal processes. Targets of Mec1 include proteins required for checkpoint arrest (Rad9, Schwartz et al. 2002, Ma et al. 2006), DNA replication (RPA, Bartrand et al. 2005), fork stability (Mrc1, Osborn \& Elledge 2003), DNA repair/recombination (Sae2, Flott \& Rouse 2005, Cartagena-Lirola et al. 2006), and chromosome structure (H2A, Downs et al. 2000). Additionally, in-vitro protein CHIP analysis identified Axl2, Hog1, and Ptk2 as high-to-moderately specific substrates of Mec1, and histone H1, Hgl1, Mek1, Rad9, Red1, Swi6, and Tub4 as low specificity substrates (Zhu et al. 2000). The nature of the mammalian ATM and
ATR targets also reflects their functions. The targets identified to date include proteins involved in checkpoint regulation (Rad9, CHEK2), DNA repair/ recombination (BRCA1, Mre11A, BLM [budding yeast Sgs1]), and chromosome structure $(\gamma \mathrm{H} 2 \mathrm{AX}$, Smc1) (Kim et al. 1999, Traven \& Heierhorst 2005).

Mammalian ATM is also a phospho-protein being phosphorylated at Ser1981 in response to DSB, stalled forks, and general changes in chromosome structure (Bakkenist \& Kastan 2003, Stiff et al. 2006). Although initially characterized as an autophosphorylation event (Bakkenist \& Kastan 2003), ATM phosphorylation at Ser1981 can also occur in ATR-dependent (and ATM-independent) manner (Stiff et al. 2006). In general, phosphorylation of ATM is thought to be essential for its activation at the cellular level. Currently there is no evidence for a similar requirement for phosphorylation of ATR, $\mathrm{Mec1}$, or Tel1 for their activation. In this regard it is noteworthy that ATM contains the highest number of SQ/TQ motifs, many of which occur in clusters. The numbers of SQ/TQ motifs present over the entire length of each protein are: ATM (28/3066), ATR (16/2635), Mec1 (9/2324), and Tel1 (12/2787).

All mecl mutations that abolish its kinase activity show a mecl-null phenotype (Paciotti et al. 2001). Similarly, over-expression of an ATR-kd (kinase dead) allele confers many defects associated with ATR inactivation (Cliby et al. 1998). These observations indicate that kinase activity is essential for their functions.

\section{Roles of Mec1 during meiosis}

Meiosis is a specialized cell division program during which a single round of genome duplication is followed by two successive rounds of chromosome segregation, resulting in the halving of the genome. The meiosis-specific part of the program is the period leading up to the first chromosome segregation during which homologs rather than sister chromatids segregate. In most organisms, including budding yeast and mammals, accurate disjunction of homologs requires the generation of hundreds of DSB catalyzed by Spo11 and the repair of these breaks using one homolog to create crossovers between each pair of homologs; such crossovers provide the necessary physical connection between each homolog pair at the time of chromosome segregation. Analogous 
to the mitotic cell cycle program, the meiotic program has the regulatory network poised to trigger arrest in response to exogenously induced DNA damage as well as aberrations in the processing of Spo11induced DSB. Based on the nature of defects responsible for triggering checkpoint response and the specific stage at which cells remain arrested, programmed arrests or delays during meiosis are referred to as 'pachytene-', 'rad50S-', 'recombination-', or 'damage-' checkpoint arrests (Hochwagen \& Amon 2006). In all cases examined, cells delay or prevent the onset of meiosis I and remain in prophase I; for simplicity we will refer all conditions that lead to programmed arrest/delay prior to meiosis I as triggering 'prophase checkpoint' when discussing meiotic checkpoint roles of Mec1/Tel1.

MECl (mitosis entry checkpoint gene 1), also known as ESRI (essential for recombination), was cloned by two independent research groups based on its roles in preventing mitosis in the presence of unrepaired DNA damage (Weinert et al. 1994), and as a gene required for conferring MMS resistance (Kato \& Ogawa 1994). Kato \& Ogawa examined the meiotic roles of ESRI/MECI and observed an induction of its expression during meiosis, reaching maximum levels (about 20 times the mitotic level) in pachytene. The esrl-1 allele isolated also exhibited defects in meiotic recombination and progression. Subsequent studies characterizing various mecl-null strains (e.g. mecld, mecl-kd, or mecl-1 [different from esrl-1] strains maintained viable by smll, a suppressor mutation) confirmed their meiotic roles, as viability of the resulting spores is reduced to 30-50\% compared with $>90 \%$ for MECl (e.g. Lydall et al. 1996, J. Carballo, N. Hashash \& R. Cha unpublished).

Since Mec1 is a central player in mediating checkpoint responses, the observed spore inviability in mecl mutants could stem from the inability to mount an appropriate 'checkpoint' response following the induction of programmed meiotic DSB. On the other hand, Mec1's primary role during the unchallenged life of the cell could be coordination of fundamental chromosomal processes (Cha \& Kleckner 2002), and mecl spore inviability could have stemmed from failure to regulate essential meiotic events. Below, we present a case for the latter possibility by reviewing the available data from the viewpoint that Mec1 may be an essential component of basic meiotic chromosomal processes.
The checkpoint role of Mec1 is also discussed, but only briefly, as an in-depth review on the matter has recently been published (Hochwagen \& Amon 2006).

\section{Mecl is an essential checkpoint regulator during challenged meiosis}

During mitotic cell cycle progression, Mec1 mediates G1/S and G2/M checkpoint arrests in response to DNA-damaging agents (Weinert et al. 1994, Siede et al. 1996). It is also required for S-phase arrest triggered by accidentally stalled replication forks (Sogo et al. 2002). Similarly Mec1 is required for the prophase arrest induced by mutations (e.g. $\operatorname{rad} 50 \mathrm{~S}$, $d m c 1 \Delta$, zipla) that affect the processing of Spo11catalyzed DSB (Lydall et al. 1996) as well as for hydroxyurea (HU)-induced meiotic S-phase checkpoint arrest (Borde et al. 2000). In all cases exam-

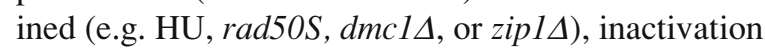
of Mec1 leads to efficient progression through meiotic divisions and generation of inviable spores (Hochwagen \& Amon 2006; Figure 1).

The nature of DNA aberrations triggering S-phase arrest in HU-treated cells is likely to be the same during both mitotic and meiotic S-phases - i.e. the persistence of RPA-coated single-stranded (ss) DNA, which results from the uncoupling of leading and lagging strand DNA synthesis on accidentally stalled replication forks (Sogo et al. 2002). The signal sensed by Mec1 in dmcl $\Delta$ mutants is also likely to be similar to that sensed during the mitotic G2/M checkpoint triggered by the presence of unrepaired DSB - i.e. the persistence of RPA-coated ssDNA following resection of the $5^{\prime}$ end of DSB (Pellicioli et al. 2001, Lisby et al. 2004). Prophase arrest in zipl $\Delta$ mutants is interesting in that the arrest occurs despite the fact that DSB disappear with an apparently normal kinetics (e.g. Borner et al. 2004). The nature of aberration responsible for triggering the checkpoint following Zip1 activation is currently unclear.

Curiously, Zip1 physically interacts with Psy2, a component of the phosphatase complex known as HTP-C (histone H2A phosphatase complex) (Ito et al. 2001). HTP-C regulates recovery from DNA damage checkpoint arrest by dephosphorylating $\mathrm{H} 2 \mathrm{~A}$, a key target of Mec1/Tel1 (Keogh et al. 2005). Similarly, dephosphorylation of $\gamma \mathrm{H} 2 \mathrm{AX}$ by protein phosphatase 2 (PP2) is required for recovery from damage checkpoint arrest in mammals (Chowdhurry et al. 2005). Involvement of PP1 
A.

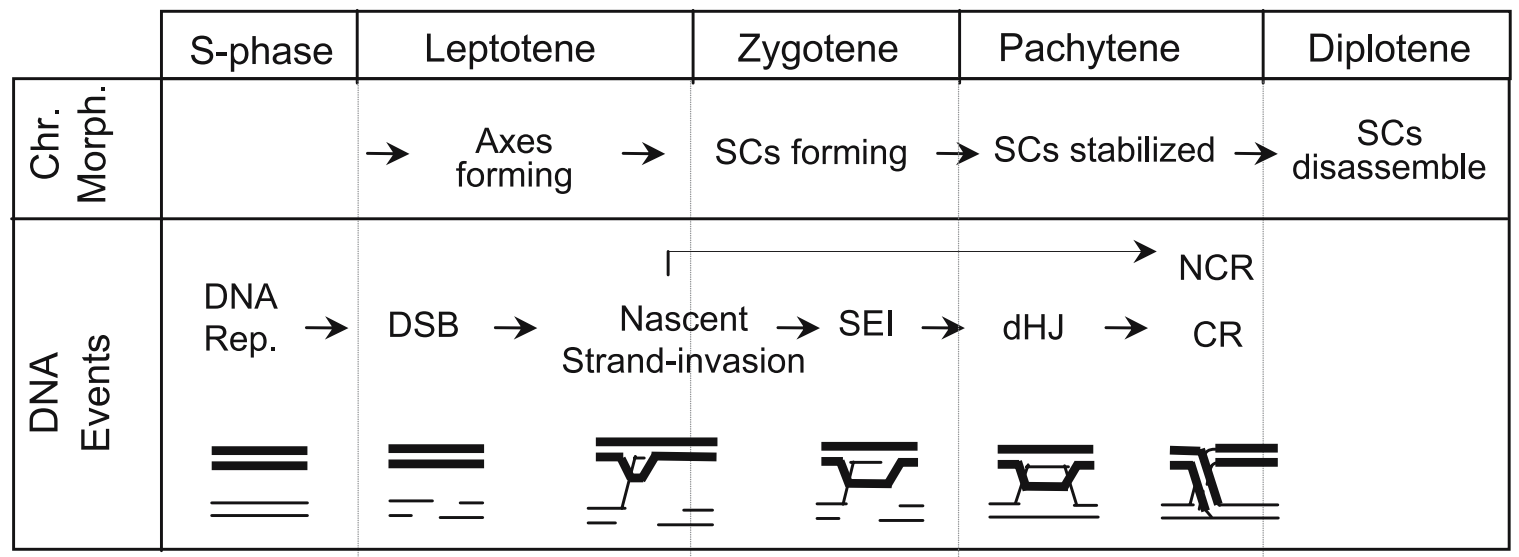

B.

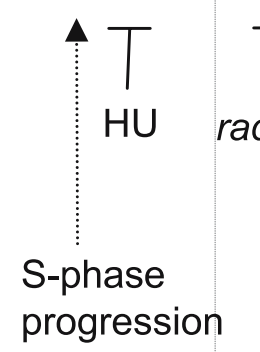

in recom

SC

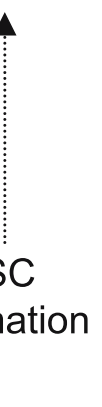

$T$

zip14

IH-bias

C.

RPA a, b, c

Sae2 a,c

Rad50, Mre11, Xrs2 a,b,d

Hop1 a, c

Figure 1. Meiotic roles of Mec1. A: DNA and chromosomal events during meiotic prophase I. Chr.Morph: chromosome morphology, CR: crossover, NCR; non-crossover, SEI: single-end invasion, dHJ: double Holiday junction, SC: synaptonemal complex. Schematically depicted in the 'DNA event' are the recombination intermediates involved in the formation of CR. Commitment to the CR or NCR pathways takes place after the nascent strand invasion step, and the majority of NCR arise independently of dHJ (e.g. Borner et al. 2004). B: Mec1 is a key

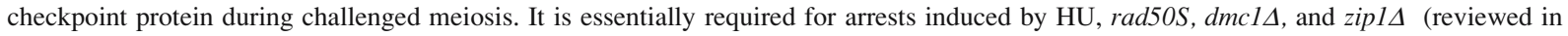
Hochwagen \& Amon 2006). During normal meiosis, Mec1 is likely to play a critical role in promoting or regulating the key meiotic chromosomal processes. The fundamentally positive nature of Mec1 function(s) during unchallenged meiosis is based on the observations that inactivation of Mec1 leads to a block in meiotic progression rather than faster transit (see text). Molecular mechanisms underlying interhomolog bias, SC development, and crossover distribution are still unclear; the positions of arrows along the meiotic time-line simply denotes Mec1's involvement in these processes rather than marking the precise execution point. C: Confirmed (or likely) meiotic targets of Mec1. The nature of evidence in each case is as indicated (a: genetics data in yeast, $\mathbf{b}$ : genetics data in mammals, $\mathbf{c}$ : biochemical data in yeast, $\mathbf{d}$ : biochemical data in mammals). The width of rectangle denotes either the period during which the function of each protein is required and/or the period during which phosphorylation of each protein is observed. 
(protein phosphatase I) in $d m c l \Delta$ checkpoint adaptation during yeast meiosis has also been reported (Hochwagen et al. 2005). Taken together, these observations raise an intriguing possibility that Zip1 (and/or the status of synaptonemal complex [SC]) may regulate checkpoint recovery during yeast meiosis. The notion that SC polymerization plays a role in sensing interchromosomal entanglements and triggering an appropriate response has been presented previously (Kleckner et al. 1991, Bishop et al. 1992).

Essential components of the Mec1/Tel1 damage checkpoint pathway include Rad24, the budding yeast '9-1-1 complex' ( $\operatorname{Rad} 17 / \mathrm{Mec} 3 / \mathrm{Ddc} 1), \operatorname{Rad} 9$, and $\operatorname{Rad} 53$. $\operatorname{Rad} 24$ and $\operatorname{Rad} 17 / \mathrm{Mec} 3 / \mathrm{Ddc} 1$ are considered to be acting upstream of Mec1, whereas Rad9 and Rad53 function as downstream effectors (Zhou \& Elledge 2000). Available evidence suggests that the upstream components are required for all Mec1/ Tel1-mediated meiotic checkpoint responses; the requirement for $\operatorname{Rad} 9$, on the other hand, appears to depend on the nature of $\operatorname{defect}(\mathrm{s})$ responsible for triggering the arrest (Hochwagen \& Amon 2006). The potential involvement of Rad53 in meiotic checkpoint regulation has not been directly examined; however, it is likely that at least some of its functions are carried out by Mek1, the meiosisspecific paralog of Rad53 (Usui et al. 2001, Hochwagen \& Amon 2006).

Dissecting the checkpoint roles of $\operatorname{Rad} 24, \operatorname{Rad} 17$, $\mathrm{Mec} 3$, and Ddc1 has been complicated by the fact that these proteins are required not only for checkpoint response signaling but also in the process of recombination or SC formation (Shinohara et al. 2003, Wu \& Burgess 2006). For instance, elimination of Rad17 leads to defective processing of meiotic DSB, which in itself triggers a checkpoint response (Wu \& Burgess 2006). It is noteworthy that meiotic phenotypes conferred by $\mathrm{mecl}$, rad24, and $\mathrm{rad} 17$ are not identical (Lydall et al. 1996, Wu \& Burgess 2006) raising the possibility that these proteins carry out functions that might be independent of one another during meiosis.

\section{Mecl functions as a positive regulator of normal meiotic progression}

Meiotic prophase (i.e. the period between the completion of the genome duplication and the first meiotic division) in budding yeast (SK1 background) is about $2 \mathrm{~h}$ long. This meiotic prophase duration of $2 \mathrm{~h}$ coincides with the time it takes to process the Spo11-induced breaks into the final crossover recombination products (e.g. Padmore et al. 1991), raising the possibility that the status of ongoing meiotic recombination is monitored, and that the onset of meiosis I is coupled to the completion of DSB repair. Given the essential nature of Mec1 in triggering prophase arrest in $d m c 1 \Delta$ and zipl $\Delta$, it is reasonable to propose that Mec1 plays similar roles during normal meiosis in monitoring the processing of Spo11-catalyzed DSB and that defects in this process lead to the observed reduction in spore viability.

If Mec1 functions as a negative regulator of meiotic progression delaying the onset of meiosis I until the completion of DSB repair, inactivation of Mec1 should lead to a faster transit through meiosis, similar to that observed in rec- mutants (above); mecl-1 smll strains, however, proceed through meiosis with WT kinetics with regard to meiotic divisions (e.g. Lydall et al. 1996, Borde et al. 2000). Although this 'unexpected' observation could be due to Tel1 compensating for Mec1's normal function, the existence of mecl alleles that confer a meiotic arrest phenotype, even in the presence of a fully functional TEL1, argues against this notion. For example, the original mutant isolated by Kato \& Ogawa, esr 1-1, exhibits a progression defect; following transfer into sporulation medium, esrl-1 cells undergo meiotic DNA replication but only $2 \%$ of the cells proceed to from spores (Kato \& Ogawa 1994). We have also independently isolated alleles of mecl that fail to form spores (R. Cha unpublished).

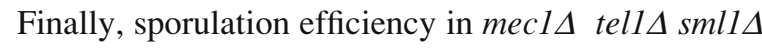
mutants is also reduced below 20\% (J. Carballo, N. Hashash \& R. Cha unpublished). This apparent failure to progress through meiosis associated with loss of Mec1 functions suggests Mec1 has a positive, rather than negative, role in promoting meiosis. Presented below are essential meiotic DNA and chromosomal processes and the potential involvement of Mec1 in each.

\section{Meitoic S-phase progression}

Meiotic S-phase is several times longer than the mitotic counterpart in all organisms examined to date. In budding yeast (SK1 background), mitotic- and meiotic-S phases are about $20 \mathrm{~min}$ and $60-80 \mathrm{~min}$, respectively (Williamson et al. 1983, Cha et al. 2000). The lengthening of S-phase during meiosis is 
proposed to reflect the extra time needed for developing additional chromosomal features that are later utilized for meiosis-specific chromosomal metabolism (Holm 1977). Consistent with this notion, the lengthening of meiotic S-phase is dependent on the establishment of DSB-independent homolog pairing mediated by Spo11 (Cha et al. 2000).

$\mathrm{Mec} 1$ is essentially required for replication fork progression through genetically specified replication slow zones (RSZ) during unchallenged mitotic S-phase (Cha \& Kleckner 2002). This essential role of Mec1 is bypassed by mutations that result in higher intracellular dNTP levels (Zhao et al. 1998, Cha \& Kleckner 2002), suggesting that Mec1 may regulate fork progression by coupling fork processivity to the availability of dNTP in the cell. With regard to a possible physiological relevance for such programmed fork slowing and the subsequent Mec1regulated progression, it was proposed that Mec1 might be playing a role in coupling ongoing DNA replication to the replication-dependent chromosome feature development - e.g. sister chromatid cohesion (Uhlmann \& Nasmyth 1998), installation of nucleosomes (Shibahara \& Stillman 1999), and meiosisspecific chromosome modification (Wu \& Lichten 1994, Ohta et al. 1998, Cha et al. 2000). More specifically, Mec1 would monitor the installation of key features in newly replicated regions, and (a) promote progression of nearby replication forks, and (b) activate late firing origins when these events have been completed.

A number of observations are consistent with the notion that Mec1 is a central regulator of the basic S-phase program. These include: (a) Rad53, a key downstream target of Mec1, interacts physically with Asf1 - Asf1 is a component of the replicationdependent chromatin remodelling complex, which is needed for proper completion of DNA synthesis during normal S phase (Le et al. 1997); (b) Mec1/ Rad53 regulates the timing of late origins activation during normal S-phase (Shirahige et al. 1998); (c) Mec1 regulates fork progression through RSZ (Cha \& Kleckner 2002); (d) Mre11, a known genetic interactor of Mec1/Tel1, and a confirmed target of ATM in mammals, promotes S-phase-specific chromatin remodeling during meiosis (Ohta et al. 1998, Kim et al. 1999, Nakada et al. 2004); and (e) the mammalian sister chromatid cohesin molecule Smc1 is a target of ATM/ATR (Kim \& Kastan 2002, Yazdi et al. 2002).
We have isolated a mecl allele that confers defects in meiotic S-phase progression (J. Cauwood \& R.

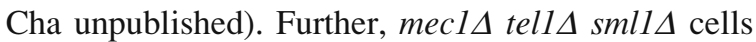
are unable to progress through meiotic S-phase (J. Carballo \& R. Cha unpublished). Since these mutants can proliferate, they presumably undergo relatively normal mitotic DNA replication. Thus, the meiotic S-phase problem in mecl mutants is unlikely to stem from a failure in regulating dNTP pools, responding to spontaneously stalled replication forks, or from other 'usual' challenges associated with the genome duplication in general. Given that additional meiosis-specific chromosomal features are laid down during meiotic S-phase (Wu \& Lichten 1994, Ohta et al. 1998, Murakami et al. 2003) and that they are required for the timely transit through S-phase, a differential requirement for $\mathrm{Mec} 1$ in meiotic S-phase progression may reflect its involvement in these processes. It will be interesting to examine potential genetic interactions between mecl and meiotic chromosomal proteins known to affect meiotic S-phase progression - i.e. Spo11, Rec8, and Mre11 - in regard to meiotic S-phase progression and establishment of replication-dependent chromosome features.

\section{Meiotic recombination}

The original esr $1-1$ allele confers a reduction in both intra- and inter-genic meiotic recombinational events (Kato \& Ogawa 1994). In budding yeast, such overall deficit in meiotic recombination could stem from either the reduction in the amount of breaks generated and/or failure to process DSB into recombination products. For instance, the recombination defect in hop $1 \Delta$, redl $1 \Delta$ or mekl $\Delta$ is accompanied by a significant reduction in the amount of breaks generated (e.g. Schwacha \& Kleckner 1997, Xu et al. 1997), whereas that observed in mutations in the RAD52 epistasis group stems from defects in processing Spo11-catalyzed DSB (e.g. Schwacha \& Kleckner 1997). Currently available evidence points to the latter possibility as a mechanism underlying the observed esrl-1 phenotype; a potential link between Mec1(Tel1) and DSB formation has not yet been reported.

Although the context in which meiotic breaks arise (i.e. programmed vs accidental) and the specific mechanisms involved are different, the basic mechanisms of repairing DSB are conserved during mitotic and meiotic programs (Paques \& Haber 
1999). In general, all of the players in mitotic DSB repair participate in the repair of meiotic DSB, but with a number of meiosis-specific adaptations that facilitate the eventual formation of crossovers between homologs (e.g. Kleckner 1996).

The repair of Spo11-catalyzed DSB begins with the enzymatic removal of Spo11 from the break ends followed by nucleolytic resection of the $5^{\prime}$-strand to generate 3 '-single-stranded tails (Neale et al. 2005). These tails are coated first by RPA, followed by Rad51 and Dmc1 (a meiosis-specific RecA protein) and participate in the homology search and strand invasion (Paques \& Haber 1999). Many proteins become localized to the site of breaks including Mre11 and the rest of the recombinational repair machinery (e.g. RAD52 epistasis group) (Lisby et al. 2004). A number of chromosome structural and repair proteins become phosphorylated during this process (Downs et al. 2004, Cartagena-Lirola et al. 2006). Since Mec1 (or ATM/ATR) plays a central role in the localization and phosphorylation of many of these proteins (e.g. RPA, H2A, Mre11, Smc1, and Rad55) the observed reduction in meiotic recombination could easily be due to a failure to promote (early steps of) DSB repair.

\section{Inter-homolog bias}

In contrast to mitotic recombination where DSB are repaired utilizing a sister chromatid, meiotic recombination takes place between homologs. In budding yeast the repair of meiotic DSB occurs with an estimated 5-fold bias toward inter-homolog events (Schwacha \& Kleckner 1997). Although the molecular mechanism underlying establishment and enforcement of the inter-homolog bias is unclear, proteins involved in recombination (e.g. Dmc1, Mer3, and Msh5), chromosome structure (e.g. Hop1, Red1, and Zip1), and a regulatory kinase (Mek1) have been shown to be required (Schwacha \& Kleckner 1997, Niu et al. 2005).

In mecl-1 smll mutants, recombination events between homologs are observed at reduced rates; this reduction is accompanied by an increase in ectopic recombination. The observed loss of inter-homolog bias is not a secondary effect of inappropriate progression (e.g. compromising inter-homolog recombination by premature separation of homologous chromosomes), but is likely to reflect Mec1's involvement in regulating recombination partner choice (Grushcow et al. 1999). Thompson \& Stahl also isolated $\mathrm{Mec1}$ as a protein whose inactivation leads to increased recombination between sister chromatids during meiosis, presumably at the expense of inter-homolog events (Thompson \& Stahl 1999).

Recently, we identified Hop1 as a meiosis-specific target of Mec1 (J. Carballo, A. Johnston \& R. Cha unpublished). Hop1 is an evolutionarily conserved protein required for multiple meiotic processes. One of its functions, together with Red1 and Mek1, is to ensure that meiotic DSB are repaired via the Dmc1mediated (i.e. inter-homolog) pathway (Schwacha \& Kleckner 1997, Niu et al. 2005). Dmc1 is an evolutionarily conserved meiosis-specific RecA homolog and is essential for meiotic inter-homolog recombination (Bishop et al. 1992, Gasior et al. 2001). Thus, characterization of Mec1/Tel1 dependent phosphorylation of Hop1 may provide a plausible mechanism by which Mec1 participates in enforcing inter-homolog bias.

\section{Homolog synapsis}

The synaptonemal complex (SC), a proteinaceous structure, comprising two lateral elements and a central element, mediates the intimate synapsis between the homologs during meiotic prophase I. $\mathrm{SC}$ formation begins during the leptotene to zygotene transition, is completed by pachytene, and becomes dissolved by diplotene (Figure 1). The SC is observed in most organisms including budding yeast, plants, worms, and mammals, where intimate homolog synapsis mediated by the SC is essential for normal meiosis (Page \& Hawley 2004, Zickler 2006). Status of SC development can be assessed by immunolocalization of either the central or the lateral components of the SC; in budding yeast, Zip1, a component of the central element, is typically utilized.

In yeast, one manifestation of aberrant SC development is the appearance of large structures called polycomplexes (PC), which stain brightly with antiZip1 antibody. PC appear in addition to or instead of the extended structures typical of normal meiosis, especially in mutants with defects in synapsis. As expected, eliminating genes that encode for the lateral (e.g. Red1 and Hop1) components of the SC leads to the appearance of extensive PC. Additional proteins required for normal SC development include those involved in (a) basic chromosome structure 
(e.g. Rec8), (b) the generation of DSB (e.g. Spo11), and (c) the processing of meiotic DSB (e.g. Dmc1, Msh5) (Page \& Hawley 2004, Zickler 2006).

Inactivation of Mec1 has been linked to the formation of PC (Grushcow et al. 1999). Although this could be an indirect consequence of an earlier problem (e.g. in recombination), it is also possible that Mec1 plays a more direct role in SC development by phosphorylating a relevant chromosome structure protein(s). The observations that (a) Mec1/ ATM/ATR interact both genetically and physically with chromosome structure proteins (e.g. Asf1, Smc1, CAF, and H2A; reviewed in Koundrioukoff et al. 2004); and (b) ATM can be activated by conditions that alter chromatin structure in the absence of DNA damage (Bakkenist \& Kastan 2003) highlight a potential interplay between chromosome structure and ATM/ATR family proteins. Also relevant in this regard is our recent identification of Hop1 as a Mec1 target (J. Carballo, A. Johnson \& R. Cha unpublished). As mentioned above, Hop1 is essentially required for SC formation, being a component of the lateral element. An attractive possibility would be that Mec1 participates in SC development by regulating Hop1 activity.

\section{Regulation of crossover distribution}

In budding yeast, meiotic recombination begins with the formation of approximately 300 Spo11-catalyzed DSB; at the end of the process each pair of homologs ends up with at least one but no more than a few crossovers. A similar numerical relationship between the number of breaks and crossovers exists for meiotic recombination in mammals. In many organisms, including budding yeast, worms, fruit flies, and mammals, the distribution of crossovers in the genome is highly regulated in that: (a) every pair of homologs acquires at least one crossover, and (b) when two or more crossovers occur between the same pair of homologs, adjacent ones are located further apart than expected from a random distribution (e.g. Jones 1987, Carpenter 1988). These two features of crossover distribution are referred to as 'obligatory crossover' and 'crossover interference', respectively. The molecular mechanisms governing the distribution of crossovers are not known. However, mutations that confer defects in chromosome structure development, recombination, and homolog pairing have been shown to weaken crossover interference.
Involvement of Mec1 in crossover distribution has been examined by Bartrand et al. (2005) where the authors investigated the consequences of altering Mec1-dependent phosphorylation sites in RPA. The RPA serine-to-aspartate (S122D) mutation significantly altered the pattern of meiotic crossover events for certain genetic intervals, suggesting that Mec1mediated RPA phosphorylation can influence the mechanism of crossing over during meiosis in a region-specific manner (Bartrand et al. 2005). Another yeast gene shown to affect meiotic crossover recombination is Sgs1, a RecQ helicase homolog. Inactivation of $\mathrm{Sgs} 1$ leads to a moderate to a dramatic increase in crossover recombination events depending on the strain background, indicating that Sgs1 has potent meiotic anti-crossover activity (Rockmill et al. 2003, Jessop et al. 2006). Interestingly, the mammalian homolog, BLM, is a confirmed target of ATM (Beamish et al. 2002). In yeast, Mec1 and $\mathrm{Sgs} 1$ function synergistically in suppressing gross chromosomal aberrations (Cobb et al. 2005), and $\mathrm{Mec} 1$ is required for the localization of Sgs1 to a damage-induced multi-protein complex (Chiolo et al. 2005). Further, a few of the proteins involved in crossover regulation contain an unusually high number of SQ/TQ motifs including Msh5 (8/901) and Exo1 (10/702). It will be interesting to examine whether they are targets of Mec1 and, if so, whether Mec1-dependent phosphorylation regulates crossover distribution. Finally, it is noteworthy that one of the original defects reported for the fly mei-41 mutant is defects in crossover regulation (Carpenter 1979).

\section{Summary}

Here, we presented meiotic defects of mecl mutants from the perspective that the observed phenotype stems from failure to regulate and promote essential chromosomal events. The complex nature of the mecl phenotype suggests that its function(s) is likely to be required for a number of different meiotic processes such as S-phase progression, recombination, inter-homolog bias, and chromosome synapsis (Figure 1). Although a number of Mec1/Tel1 and ATM/ATR targets have been identified, the focus thus far has been on those involved in checkpoint regulation and DNA repair in response to exogenously induced DNA damage. Identification and characterization of meiosis-specific targets of Mec1/ 
Tel1 during normal meiotic progression should provide invaluable information regarding Mec1's essential meiotic function(s).

\section{References}

Bakkenist C, Kastan M (2003) DNA damage activates ATM through intermolecular autophosphorylation and dimer dissociation. Nature 421: 499-506.

Barlow C, Hirotsune S, Paylor R et al. (1996) Atm-deficient mice: a paradigm of ataxia telangiectasia. Cell 86: 159-171.

Bartrand A, Iyasu D, Marinco S, Brush G (2005) Evidence of meiotic crossover control in Saccharomyces cerevisiae through Mec1-mediated phosphorylation of replication protein A. Genetics 172: 27-39.

Beamish H, Kedar P, Kaneko H et al. (2002) Functional link between BLM defective in Bloom's Syndrome and the Ataxiatelengiectasia- mutated Protein ATM. J Biol Chem 277: 30515.

Bishop D, Park D, Xu L, Kleckner N (1992) DMC1: A meiosisspecific yeast homolog of E. coli recA required for recombination, synaptonemal complex formation, and cell cycle progression. Cell 69: 439-458.

Borde V, Goldman A, Lichten M (2000) Direct coupling between meiotic DNA replication and recombination initiation. Science 290: 806-809.

Borner G, Kleckner N, Hunter N (2004) Crossover/noncrossover differentiation, synaptonemal complex formation, and regulatory surveillance at the leptotene/zygotene transition of meiosis. Cell 117: 29-45.

Brown E, Baltimore D (2000) ATR disruption leads to chromosomal fragmentation and early embryonic lethality. Genes Dev 14: $397-402$.

Carpenter A (1979) Recombination nodules and synaptonemal complex in recombination-defective females of Drosophila melanogaster. Chromosoma 75: 259-292.

Carpenter A (1988) Thoughts on Recombination Nodules, Meiotic Recombination, and Chiasmata. Washington, DC: American Society of Microbiology.

Cartagena-Lirola H, Guerini I, Viscardi V, Lucchini G, Longhese M (2006) Budding yeast Sae2 is an in vivo target of the Mec1 and Tel1 checkpoint kinases during meiosis. Cell Cycle 5: 1549-1559.

Cha R, Kleckner N (2002) ATR homolog Mec1 promotes fork progression, thus averting breaks in replication slow zones. Science 297: 602-606.

Cha R, Weiner B, Keeney S, Dekker J, Kleckner N (2000) Progression of meiotic DNA replication is regulated by interchromosomal interaction proteins, negatively by Spo11p and positively by Rec8p. Genes Dev 14: 493-503.

Chiolo I, Carotenuto W, Maffioletti G, Petrini J, Foiani M, Liberi G (2005) Srs2 and Sgs1 helicases associate with Mre11 in different subcomplexes following checkpoint activation and CDK1-mediated Srs2 phosphorylation. Mol Cell Biol 25: 5738-5751.

Chowdhurry D, Keogh M-C, Ishii H, Peterson C, Suratowski S, Lieberman J (2005) g-H2AX dephosphorylation by protein phosphatase 2A facilitates DNA double-strand break repair. Mol Cell 20: 801-809.

Cliby W, Roberts C, Cimprich K et al. (1998) Overexpression of a kinase-inactive ATR protein causes sensitivity to DNA-damaging agents and defects in cell cycle checkpoints. EMBO $J \mathbf{1 7}$ : $159-160$

Cobb J, Schleker T, Rohas V, Bjergbaek L, Tercero J, Gasser S (2005) Replisome instability, fork collapse, and gross chromosomal rearrangements arise synergistically from Mec1 kinase and RecQ helicase mutations. Genes Dev 19: 3055-3069.

Cuadrado M, Martinez-Pastor B, Murga M et al. (2006) ATM regulates ATR chromatin loading in response to DNA doublestrand breaks. J Exp Med 203: 297-303.

Downs J, Lowndes N, Jackson S (2000) A role for Saccharomyces cerevisiae histone H2A in DNA repair. Nature 408: 1001-1004.

Downs J, Allard S, Johns-Robitaille O et al. (2004) Binding of chromatin-modifying activities to phosphorylated histone $\mathrm{H} 2 \mathrm{~A}$ at DNA damage sites. Mol Cell 16: 979-990.

Flott S, Rouse J (2005) Slx4 becomes phosphorylated after DNA damage in a Mec1/Tel1-dependent manner and is required for repair of DNA alkylation damage. Biochem $J$ 391: 325-333.

Gasior S, Olivares H, Hari D, Weichselbaum R, Bishop D (2001) Assembly of RecA-like recombinases: distinct roles for mediator proteins in mitosis and meiosis. Proc Natl Acad Sci USA 98: 8411-8418.

Greenwell PW, Kronmal SL, Porter SE, Gassenhuber J, Obermaier B, Petes TD (1995) TEL1, a gene involved in controlling telomere length in S. cereviseae, is homologous to the human ataxia telangiectasia gene. Cell 82: 823-829.

Grushcow JM, Holzen TM, Park KJ, Weintert T, Licheten M, Bishop DK (1999) Saccharomyces cerevisiae checkpoint genes MEC1, RAD17 and RAD24 are required for normal meiotic recombination partner choice. Genetics 153: 607-620.

Hochwagen A, Amon A (2006) Checking your breaks: surveillance mechanism for meiotic recombination. Curr Biol 16: R217-228.

Hochwagen A, Tham W, Brar G, Amon A (2005) The FK506 binding protein Fpr3 counteracts protein phosphatase 1 to maintain meiotic recombination checkpoint activity. Cell 122: 861-873.

Holm PB (1977) The premeiotic DNA replication of euchromatin and heterochromatin in Lilium longiflorum (Thunb.). Carlsberg Res Commun 42: 249-281.

Ito T, Chiba T, Ozawa R, Yoshida M, Hattori M, Sakaki Y (2001) A comprehensive two-hybrid analysis to explore the yeast protein interactome. Proc Natl Acad Sci USA 98: 4569-4574.

Jazayeri A, Falck J, Lukas C et al. (2006) ATM- and cell cycledependent regulation of ATR in response to DNA double-strand breaks. Nat Cell Biol 8: 37-45.

Jessop L, Rockmill B, Roeder G, Lichten M (2006) Meiotic chromosome synapsis-promoting proteins antagonize the anticrossover activity of Sgs1. PLoS Genet 2: e155.

Jones G (1987) Chiasmata. New York: Academic Press.

Kato R, Ogawa H (1994) An essential gene, ESR1, is required for mitotic cell growth, DNA repair and meiotic recombination in Saccharomyces cerevisiae. Nucleic Acids Res 22: 3104-3112.

Keogh M-C, Kim J, Downey M et al. (2005) A phosphatase complex that dephosphorylates $\mathrm{gH} 2 \mathrm{AX}$ regulates DNA damage checkpoint recovery. Nature 439: 497-501. 
Kim S-T, Kastan M (2002) Involvement of the cohesin protein, Smc1, in Atm-dependent and independent responses to DNA damage. Genes Dev 16: 560-570.

Kim S-T, Lim D-S, Canman C, Kastan M (1999) Substrate specificities and identification of putative substrates of ATM kinase family members. J Biol Chem 274: 37538-37543.

Kleckner N (1996) Meiosis: how could it work? Proc Natl Acad Sci USA 93: 8167-8174.

Kleckner N, Padmore R, Bishop DK (1991) Meiotic chromosome metabolism: one view. Cold Spring Harbor Symp Quant Biol 56: 729-743.

Koundrioukoff S, Polo S, Almouzni G (2004) Interplay between chromatin and cell cycle checkpoints in the context of ATR/ ATM-dependent checkpoints. DNA Repair 3: 969-978.

Le S, Davis C, Konopka J, Sternglanz R (1997) The new S-phasespecific genes from Sacchaomyces cerevisiae. Yeast 13: 1029-1042.

Lisby M, Barlow J, Burgess R, Rothstein R (2004) Choreography of the DNA damage response: spatiotemporal relationships among checkpoint and repair proteins. Cell 118: 699-713.

Lydall D, Nikolsky Y, Bishop DK, Weinert T (1996) A meiotic recombination checkpoint controlled by mitotic checkpoint genes. Nature 383: 840-843.

Ma J, Lee S, Duong J, Stern D (2006) Activation of the checkpoint kinase Rad53 by the phosphatidyl inositol kinase-like kinase Mec1. J Biol Chem 281: 3954-3963.

Mallory J, Bashkirov V, Trujilo K et al. (2003) Amino acid changes in Xrs2p, Dun1p, and Rfa2p that remove the preferred targets of the ATM family of protein kinases do not affect DNA repair of telomere length in Saccharomyces cerevisiae. DNA Repair 2: 1041-1064.

McGowan C, Russell P (2004) The DNA damage response: sensing and signaling. Curr Opin Cell Biol 16: 629-633.

Melo J, Cohen J, Toczyski D (2001) Two checkpoint complexes are independently recruited to sites of DNA damage in vivo. Genes Dev 15: 2809-2821.

Morrow D, Tagle D, Shiloh Y, Collins F, Hieter P (1995) TEL1, an $S$. cerevisiae homolog of the human gene mutated in ataxia telangiectasia, is functionally related to the yeast checkpoint gene MEC1. Cell 82: 831-840.

Murakami H, Borde V, Shibata T, Lichten M, Ohta K (2003) Correlation between premeiotic DNA replication and chromatin transition at yeast recombination initiation sites. Nucleic Acids Res 31: 4085-4090.

Nakada D, Hirano Y, Sugimoto K (2004) Requirement of the Mre11 complex and exonuclease 1 for activation of the Mec1 signalling pathway. Mol Cell Biol 24: 10016-10025.

Neale M, Pan J, Keeney S (2005) Endonucleolytic processing of covalent protein-linked DNA double-strand breaks. Nature 436: 1053-1057.

Niu H, Wan L, Baumgartner B, Schaefer D, Loidl J, Holligsworth N (2005) Partner choice during meiosis is regulated by Hop1 promoted dimerization of Mek1. Mol Biol Cell 16: 5804-5818.

Ohta K, Nicola A, Furuse M, Nabetani A, Ogawa H, Shibata T (1998) Mutations in the MRE11, RAD50, XRS2, and MRE2 genes alter chromatin configuration at meiotic DNA doublestranded break sites in premtiotic and meiotic cells. Proc Natl Acad Sci USA 95: 646-651.
Osborn A, Elledge S (2003) Mrc1 is a replication fork component whose phosphorylation in response to DNA replication stress activates Rad53. Genes Dev 17: 1755-1767.

Paciotti V, Clerici M, Scotti M, Lucchini G, Longhese M (2001) Characterization of mec1 kinase-deficient mutants and of new hypomorphic mecl alleles impairing subsets of the DNA damage response pathway. Mol Cell Biol 21: 3913-3925.

Padmore R, Cao L, Kleckner N (1991) Temporal comparison of recombination and synaptonemal complex formation during meiosis in S. cerevisiae. Cell 66: 1239-1256.

Page S, Hawley R (2004) The genetics and molecular biology of the synaptonemal complex. Annu Rev Cell Dev Biol 20: 525-558.

Paques F, Haber JE (1999) Multiple pathways of recombination induced by double-strand breaks in Saccharomyces cerevisiae. Micro Molec Biol Rev 63: 349-404.

Pellicioli A, Lee S, Lucca C, Foiani M, Haber J (2001) Regulation of Saccharomyces Rad53 checkpoint kinase during adaptation from DNA damage-induced G2/M arrest. Mol Cell 7: 293-300.

Perry J, Kleckner N (2003) The ATRs, ATMs, and TORs are giant HEAT repeat proteins. Cell 112: 151-155.

Rockmill B, Fung J, Branda S, Roeder G (2003) The Sgs1 helicase regualtes chromosome synapsis and meiotic crossing over. Curr Biol 13: 1954-1962.

Sanchez Y, Desany B, Jones W, Liu Q, Wang B, Elledge S (1996) Regulation of RAD53 by the ATM-like kinases MEC1 and TEL1 in yeast cell cycle checkpoint pathways. Science 271: 357-360.

Schwacha A, Kleckner N (1997) Interhomolog bias during meiotic recombination: meiotic functions promote a highly differentiated interhomolog-only pathway. Cell 90: 1123-1135.

Schwartz M, Doung J, Sun Z, Morrow J, Pradhan D, Stern D (2002) Rad9 phosphorylation sites couple $\operatorname{Rad} 53$ to the Saccharomyces cerevisiae DNA damage checkpoint. Mol Cell 9: $1055-1065$.

Shibahara K, Stillman B (1999) Replication-dependent marking of DNA by PCNA facilitates CAF-1-coupled inheritance of chromatin. Cell 96: 575-585.

Shinohara M, Kazuko S, Ogawa T, Shinohara A (2003) The mitotic DNA damage checkpoint protein $\operatorname{Rad} 17$ and $\operatorname{Rad} 24$ are required for repair of double-strand breaks during meiosis in yeast. Genetics 164: 855-865.

Shirahige K, Hori Y, Shiraishi K et al. (1998) Regulation of DNAreplication origins during cell-cycle progression. Nature 395 : 613-621.

Siede W, Allen J, Elledge S, Friedberg E (1996) The Saccharomyces cerevisiae MEC1 gene, which encodes a homolog of the human ATM gene product, is required for G1 arrest following radiation treatment. J Bacteriol 178: 5841-5843.

Sogo J, Lopes M, Foiani M (2002) Fork reversal and ssDNA accumulation at stalled replication forks owing to checkpoint defects. Science 297: 599-602.

Stiff T, Walker S, Cerosaletti K et al. (2006) ATR-dependent phosphorylation and activation of ATM in response to UV treatment or replication fork stalling. EMBO J 25: 5775-5782.

Thompson D, Stahl F (1999) Genetic control of recombination partner preference in yeast meiosis: isolation and characterization of mutants elevated for meiotic unequal sister-chromatid recombination. Genetics 153: 621-641. 
Traven A, Heierhorst J (2005) SQ/TQ cluster domains: concentrated ATM/ATR kinase phosphorylation site regions in DNAdamage-response proteins. BioEssays 27: 397-407.

Uhlmann F, Nasmyth K (1998) Cohesion between sister chromatids must be established during DNA replication. Curr Biol 8: 1095-1101.

Usui T, Ogawa H, Petrini J (2001) A DNA damage response pathway controlled by Tel1 and the Mre11 complex. Mol Cell 7: 1255-1266.

Weinert TA, Kiser GL, Hartwell LH (1994) Mitotic checkpoint genes in budding yeast and the dependence of mitosis on DNA replication and repair. Genes Dev 8: 652-665.

Williamson DH, Johnston LH, Fennell DJ, Simchen G (1983) The timing of the $\mathrm{S}$ phase and other nuclear events in yeast meiosis. Exp Cell Res 145: 209-217.

Wu H, Burgess S (2006) Two distinct surveillance mechanisms monitor meiotic chromosome metabolism in budding yeast. Curr Biol 16: 2473-2479.

Wu T-C, Lichten M (1994) Meiosis-induced double-strand break sites determined by yeast chromatin structure. Nature 263: 515-518.
Xu Y, Ashley T, Brainerd EE, Bronson RT, Meyn MS, Baltimore D (1996) Targeted disruption of ATM leads to growth retardation, chromosomal fragmentation during meiosis, immune defects, and thymic lymphoma. Genes Dev 10: 2411-2422.

Xu L, Weiner BM, Kleckner N (1997) Meiotic cells minotor the status of the interhomolog recombination complex. Genes Dev 1: $106-118$.

Yazdi P, Wang Y, Zhao W, Patel N, Lee Y-H, Qin J (2002) SMC1 is a downstream effector in the ATM/NBS1 branch of the human S-phase checkpoint. Genes Dev 16: 571-582.

Zhao X, Muller EGD, Rothstein R (1998) A suppressor of two essential checkpoint genes identifies novel protein that negatively affects dNTP pools. Mol Cell 2: 329-340.

Zhou BB, Elledge SJ (2000) The DNA damage response: putting checkpoints in perspective. Nature 408: 433-439.

Zhu H, Klemic J, Chang S et al. (2000) Analysis of yeast protein kinases using protein chips. Nat Gen 26: 283-289.

Zickler D (2006) From early homologue recognition to synaptonemal complex formation. Chromosoma 115: 158-174. 